\section{Intra-uterine Meconium Peritonitis}

Sir,

Meconium peritonitis is an aseptic chemical peritonitis, which results from perforation of the gut in utero. ${ }^{1}$ Its incidence is 1 in 35,000 live births. Meconium peritonitis can be classified into three types, i.e. generalised, cystic, and fibro-adhesive. ${ }^{2}$

Its pathogenesis involves antenatal perforation, owing to intestinal ischemia due to obstructive causes such as volvulus, atresia, stenosis, intussusception, congenital bands, meconium plugs, Hirschsprung's disease and other causes such as Meckel's diverticulum, internal hernia, viral infections (CMV and Parvovirus B19), and cystic fibrosis. Meconium itself is a very strong proinflammatory substance. When in utero intestinal perforation occurs due to one cause or the other, spilled meconium causes intra-peritoneal inflammation which subsequently leads to peritonitis. Here, we present a case of meconium peritonitis secondary to ileal atresia.

A newborn baby boy with birth weight of $3.1 \mathrm{~kg}$ was delivered through emergency lower segment cesarean section at term gestation due to fetal bradycardia. It was a booked pregnancy with no antenatal risk factor. An unremarkable anomaly scan done around 20 weeks of gestation was available. The primi gravida landed in emergency one week prior to the expected date due to decreased fetal movements. At birth, baby had poor APGAR of 5 and 8 at 1 and 5 minutes, respectively. He was resuscitated with ambu-bagging and oxygen support.

The baby had severe respiratory distress with respiratory rate of 78 breaths per minute, grunting and sub-costal recessions. He had massive abdominal ascites with bilateral hydrocele. His capillary blood gases showed type 2 respiratory failure for which he was placed on ventilatory support. C-reactive protein at birth was markedly high upto $55 \mathrm{mg} / \mathrm{dl}$. Baby's initial coagulation profile and renal function tests were also deranged. Abdominal X-ray showed paucity of gas shadows with intra-peritoneal calcifications (Figure 1). There was no meconium in anorectal canal on digital rectal examination. Ultrasound of abdomen showed massive abdomino-pelvic ascites with no visceromegaly and normal sized kidneys. TORCH screen was negative.

The baby was kept NPO on intravenous fluids and broad spectrum antibiotics according to unit protocol. Since baby's clinical presentation and abdominal X-ray

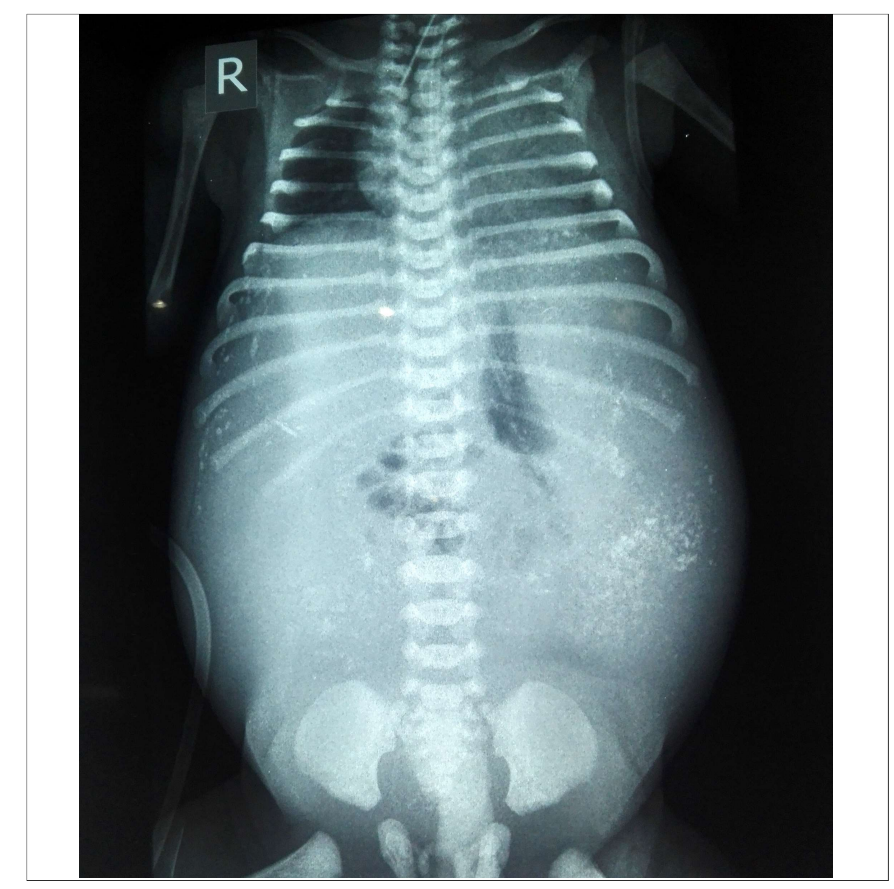

Figure 1: Preoperative and intraoperative images.

favoured a clinical diagnosis of peritonitis, we proceeded for diagnostic ascitic tap, which was found to be meconium stained.

Management of meconium peritonitis usually involves abdominal decompression through drainage and immediate surgical intervention with stoma creation or primary anastomosis. In this case, paediatric surgeon immediately proceeded for exploratory laparotomy. Peroperatively, distal ileal perforation was found with whole gut loop enclosed in a ball of meconium cyst, so distal gut loop ileostomy was made. But the baby could not survive and died on the fourth postoperative day due to complication of sepsis.

Eighty-six percent of cases with meconium peritonitis may have intra-peritoneal calcifications. ${ }^{3}$ Prognosis is good, if diagnosed in antenatal period followed by immediate intervention at birth. Overall survival rate in recent studies is around $85-90 \% .{ }^{4}$ Surgical intervention within the first 24 hours of life is advisable. The initial CRP values and postnatal cardiopulmonary insufficiency also decide the survival rate of babies with meconium peritonitis. Meconium plug syndrome and meconium ileus are important differentials, which can be ruled out by absence of extra-luminal spread of meconium.

\section{REFERENCES}

1. Lorimer WS Jr, Ellis DG. Meconium peritonitis. Surgery 1966; 60:470-5.

2. Uchida K, Koike $\mathrm{Y}$, Matsushita K. Meconium peritonitis: 
Prenatal diagnosis of a rare entity and postnatal management. Intractable Rare Dis Res 2015; 4:93-7.

3. Nam SH, Kim SC, Kim DY. Experience with meconium peritonitis. J Pediatr Surg 2007; 42:1822-5.

4. Valladares E, Rodríguez D, Vela A, Cabré S, Lailla JM. Meconium pseudocyst secondary to ileum volvulus perforation without peritoneal calcification: a case report. J Med Case Rep 2010; 4:292.
Raazia Nawaz, Arshad Khushdil and Naveed Ahmed

Department of Paediatric, Military Hospital, Rawalpindi, Pakistan

Correspondence: Dr. Arshad Khushdil, Department of

Paediatric, Military Hospital, Rawalpindi, Pakistan

E-mail: drarshad104589@yahoo.com

Received: December 14, 2017; Accepted: September 19, 2018. .......... 